\title{
General approach to risk optimisation of road bridges exposed to accidental situations
}

\author{
M. Sykora ${ }^{1}$, M. Holicky ${ }^{1} \&$ P. Maňas ${ }^{2}$ \\ ${ }^{I}$ Klokner Institute, Czech Technical University in Prague, Czech Republic \\ ${ }^{2}$ Faculty of Military Technology, University of Defence, Czech Republic
}

\begin{abstract}
Road bridges in accidental situations represent the most vulnerable components of transportation networks. Hazard situations may be caused by natural or manmade hazards such as floods, terrorist attacks or impacts of vehicles. Decisions concerning selection of protective measures can be based on the probabilistic optimisation of expected costs. In general, total structural costs consist of initial cost and consequences due to structural damage and failure. The initial cost comprises of construction cost including cost of protective measures; the failure consequences account for direct costs related to structural failure and indirect costs due to malfunction of transportation network. The paper proposes a general framework for the risk assessment based on Bayesian (causal) networks. The cost optimisation requires data on probability of occurrence and magnitude of selected accidental actions that often need to be based on expert assessments and judgements only. The present study indicates that the probabilistic cost optimisation in conjunction with Bayesian networks provide an effective tool for decisions concerning appropriate protective measures for bridges endangered by various types of hazards.
\end{abstract}

Keywords: risk optimisation, road bridges, accidental situations.

\section{Introduction}

The European road network has a vital importance for the European economy. Even smaller disruptions due to traffic restrictions or failure of some elements of the network may result in high consequences and negative environmental impacts [1]. In such a case the network and its major components are classified 
as a "critical infrastructure (object)" that can be defined as (see the European Directive 2008/114/EC [2]):

an asset, system or part thereof which is essential for the maintenance of vital societal functions, health, safety, security, economic or social well-being of people, and the disruption or destruction of which would have a significant impact in a state as a result of the failure to maintain those functions.

The Communication on a European Programme for Critical Infrastructure Protection [3] indicates that the protection of critical infrastructure is to be based on an all-hazards approach; recognising the threat from terrorism as a priority. Cost-benefit and other risk studies are deemed to be useful particularly for low probability-high consequence events where public safety is a key criterion for decision making [4].

Bridges are perceived as the most vulnerable components of the road network [5]. While single bridges can fail under particular structural damage or loads, the overall network performance is more likely to be affected by events that involve all the bridges of the network, for example by earthquakes.

In this study assessments of individual bridges are discussed from a general point of view. Methodology of the risk optimisation based on Bayesian network, applied previously for analyses of flooding [6] is used for road bridges exposed to accidental situations.

It is noted that the terminology accepted in Eurocodes - European documents for structural design [7, 8] and other European documents [2, 3] is accepted in this paper.

\section{Identification of possible critical bridges}

The following aspects should be taken into account when selecting critical bridges [9]:

- Location in urban environment,

- Conventional construction type,

- Possible traffic bottleneck formation,

- Large bridge over the sea,

- Landmark bridge,

- Very well-known bridge,

- Hard to rebuild bridge,

- Small bridge but important due to traffic volume.

Detailed procedure for the identification of possible critical bridges is provided in [9]. The failure consequences can be seen as a good indicator of the importance of a bridge [1]. 


\section{Principles of risk optimisation}

In general, risk analysis aims at consideration of all possible events together with their unfavourable consequences. Such events are often caused by extreme hazards including flooding. Adequate hazard scenarios and relevant occurrence probabilities need to be estimated $[10,11]$, often on the basis of expert assessments and judgements.

When for mutually independent hazard situations $H_{i}$ the failure $F$ of the component given a particular situation $H_{i}$ occurs with the conditional probability $\mathrm{P}\left(F \mid H_{i}\right)$, then the total probability of failure $P_{\mathrm{f}}$ is given by the law of total probability as:

$$
P_{\mathrm{f}}=\Sigma_{i} \mathrm{P}\left(F \mid H_{i}\right) \mathrm{P}\left(H_{i}\right)
$$

The conditional probabilities $\mathrm{P}\left(F \mid H_{i}\right)$ are determined by analyses of situations $H_{i}$. Annual reference period is normally accepted. The situations $H_{i}$ may lead to several events $E_{i j}$ (e.g. excessive cracking, failure of a structural member, collapse of the bridge) with adverse consequences $C_{i j}$ that are commonly expressed in monetary units. The total annual risk $R$ corresponding to the hazard situations $H_{i}$ can be expressed as:

$$
R=\Sigma_{i j} C_{i j} \mathrm{P}\left(E_{i j} \mid H_{i}\right) \mathrm{P}\left(H_{i}\right)
$$

If acceptable risk $R_{\mathrm{t}}[12]$ is specified, the bridge or its structural members can be assessed on its basis, $R<R_{\mathrm{t}}$. When the criterion of acceptable risks is not fulfilled, it is necessary to modify the system by appropriate interventions aiming at the reduction of probability of occurrence of adverse events or at the reduction of their consequences. Further information on the probabilistic risk analysis can be obtained from $[10,12,13]$.

From an economic point of view the objective is to minimize the total working-life cost of the bridge. For simplification consider hereafter assessment of the cost efficiency of a single protective measure (the presented procedure can be readily extended for decisions concerning several protective measures).

The decision parameter(s) $d$ to be optimised may represent resistance in the case of strengthening, stand-off distance, properties of energy absorbing shields etc. The parameter may be continuous, discrete or attain a single value. In general the costs of the measure consist of:

- Cost $C_{0}$ independent of the decision parameter (e.g. costs related to surveys, design, economic losses due to traffic interruptions),

- Marginal cost $C_{\mathrm{m}}$ per unit of the decision parameter.

The failure consequences $C_{\mathrm{f}}$ can be estimated on the basis of the total annual risk $R$. Since the main reason for the existence of civil infrastructures is public interest, failure consequences need to include all social losses such as (more examples in [1]): 
- Injuries and fatalities,

- Economic losses due to replacements, damaged vehicles, traffic delays, detours, economic losses of local businesses etc.,

- Environmental impacts such as air pollution, energy use or fuel consumption,

- Psychological consequences including loss of reputation or undue changes in professional practice,

- Infrastructure interdependency costs.

For consistency, all the costs need to be expressed on a common basis. Costs of the measure are normally specified in a present value. All the expected failure costs that may occur within a reference period should thus be likewise estimated in the present worth. Assuming almost independent failure events in subsequent years and small probabilities $\mathrm{P}\left(H_{i}\right)$, the expected failure cost related to a reference period $t_{\text {ref }}$ (in years) can be estimated as [14]:

$$
\mathrm{E}\left[C_{\mathrm{f}}\left(t_{\mathrm{ref}}, d\right)\right] \approx R(d)\left[1-1 /(1+q)^{t \mathrm{ref}}\right] /[1-1 /(1+q)]=R(d) Q\left(q, t_{\mathrm{ref}}\right)
$$

where $q=$ annual discount rate (e.g. 0.03, an average long-run value of the real annual discount rate in European countries); and $Q=$ time factor. The expected total costs $C_{\text {tot }}$ can now be expressed as (omitting the symbol of expectation for convenience of notation):

Measure applied: $\quad C_{\text {tot }}\left(t_{\text {ref }} ; d\right) \approx C_{0}+C_{\mathrm{m}} d+R(d) Q\left(q, t_{\text {ref }}\right)$

No measure: $\quad C_{\mathrm{tot}}\left(t_{\mathrm{ref}}\right)=R\left(\right.$ no measure) $Q\left(q, t_{\mathrm{ref}}\right)$

From eqn. (4a) the optimum value of the decision parameter $d_{\text {opt }}$ (optimum measure) can be assessed:

$$
\operatorname{minimum}_{d} C_{\mathrm{tot}}\left(t_{\mathrm{ref}} ; d\right)=C_{\mathrm{tot}}\left(t_{\mathrm{ref}} ; d_{\mathrm{opt}}\right)
$$

No measure is accepted when the total cost according to eqn. (4b) is less than the total cost of the optimum upgrade.

Apparently a more complex cost model would consider costs of inspections, maintenance, upgrades and other life-cycle costs. However, in most cases this extension hardly affects the optimum measures mitigating risks in accidental situations.

\section{Risk optimisation using Bayesian networks}

\subsection{Advantages of Bayesian networks}

Risk of various technical systems in hazard situations can be analysed using the standard techniques such as fault trees, event trees, cause-consequence method, Bayesian (causal) networks and Petri networks [10]. Fault trees cannot directly accommodate dependent basic events, which may be a serious limitation for civil engineering applications. In principle event trees can deal with such 
dependencies; however this requires great care during event-tree construction [15]. Moreover both methods suffer from the difficulty in updating based on new information. Petri Nets provide a powerful platform, but the evaluation often takes basis in Monte Carlo simulations requiring considerable computational demands [16]. These drawbacks can be overcome by the use of Bayesian probabilistic networks with discrete nodes, supplemented by decision and utility nodes [17].

With regard to practical applications Bayesian networks facilitate [18]:

- Break down of a complex task (system of structural elements) into smaller sub-tasks (foundations, piers, super-structure) that can be analysed separately by different experts,

- Illustrative interpretation of knowledge concerning structural elements based on results of measurements and expert appraisals,

- Consideration of uncertainties in action effects, material and geometrical properties, and also in the applied theoretical models,

- Modelling of complicated dependencies amongst initiation events of actions, their effects, structural properties and effects of protective measures,

- Updating of results when new information is available.

The analysis of Bayesian network is based on the specification of conditional probabilities of nodes under assumption of information on other nodes (in the direction of casual links). The analysis is based on the concept of conditional probabilities and the theory of probability. Detail information is provided e.g. in $[10,17,19]$.

\subsection{Example of the network}

Figure 1 shows an example of the Bayesian network (influence diagram) that may provide a general basis for a particular assessment. Note that the network is significantly simplified and has to be modified to reflect specific conditions of an analysed structure and considered accidental situations. In practical applications each random node may represent a sub-system and additional utility nodes may be needed. The following nodes are included in Figure 1:

- Chance nodes - Accidental situation, Action effect, Structural damage, Geotechnical conditions, and Structural properties,

- Decision nodes - Permanent and Operational measures,

- Utility nodes - Cost of measures, Social and Economic consequences, and Total cost.

Directional arrows, interconnecting all the nodes, indicate the causal links between parent and children nodes.

Given an accidental situation, the action effects dependent on accepted protective measures may lead to structural malfunction (covered in the network by the random node Structural damage) due to damage, local failures, partial or total collapse of the bridge. . Probability of structural damage and its extent may depend on geotechnical conditions and structural properties. 


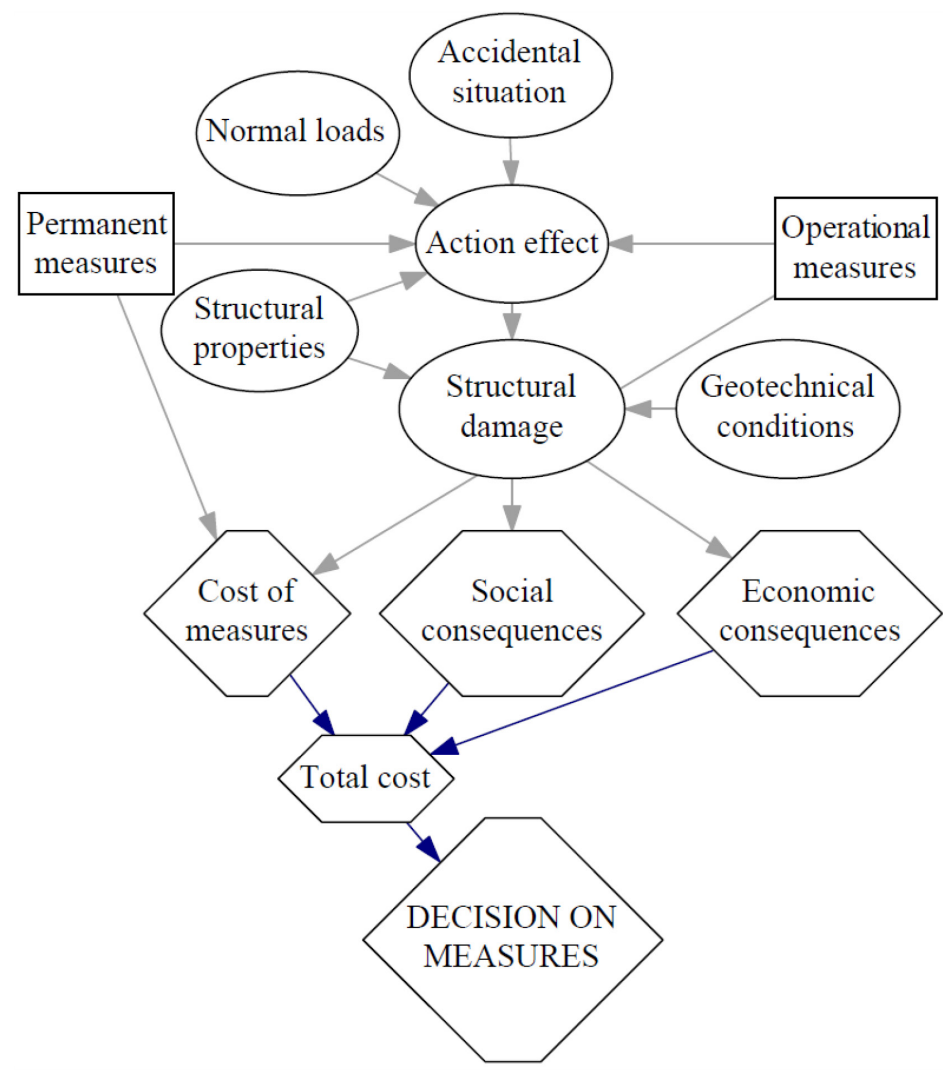

Figure 1: $\quad$ Example of the Bayesian network.

Structural damage may result in social and economic consequences. The utility node Social consequences describes the expected number of fatalities and injuries per year due to the structural collapse and a compensation cost for a fatality, which may be approximated by the so-called societal value of statistical life [19].

\section{Modelling of selected nodes}

Bridges may be exposed to the following actions [20]:

- Normal - self weight, traffic live load, thermal, wind load,

- Accidental (natural) - earthquake, landslide, hurricane and tornado, avalanche, rock fall, flood and tsunami,

- Accidental (manmade - malicious or unconscious) - explosion, fire, impact of vehicle or projectile, mining settlement,

- Human errors - design error, material flaw, construction error, misuse, lack of maintenance. 
The model of an accidental action takes into account the annual probability of occurrence of the accidental situation and the conditional probability of the accidental action (e.g. success rate in the case of blast terrorist attack). This information may be gained from experts, scenario analysis and statistical analysis of available data [21]. Limited statistical information concerning these probabilities can be found in [4].

Guidance for the development of probabilistic models of accidental action effects is provided in [22]; for instance models for earthquake, impact load and fire are discussed in [23] and blast loading in [24]. Damage on the bridge exposed to normal and accidental loads is then estimated on the basis of system modelling considering the effects of applied protective measures.

Overview of possible consequences is provided in [9]:

- Direct consequences resulting directly from the structural damage, examples include:

-- Fatalities or injuries due to structural collapse, blast pressure or secondary projectiles (concrete, steel, glass),

-- Economic losses due to cost of rehabilitation or rebuilding, cost of damaged vehicles,

- Indirect consequences describing other impacts on the society, for instance:

-- Fatalities or injuries due to malfunction of the bridge (e.g. in an emergency situation),

-- Losses due to detours, losses of local businesses affected by malfunction of the bridge,

-- Environmental and psychological impacts, infrastructure interdependency costs etc.

Indications for quantification of these consequences are provided in $[1,25]$. Permanent or operational protective measures may include [26]:

- Restriction of physical access (barriers, security officers to control access, eliminated parking near critical structures, landscaping to increase standoff distance),

- Surveillance and detection efforts (inspections, warning that the bridge is being monitored, enhanced lighting, motion sensors, removal of overgrown vegetation),

- Security planning and coordination (security plans to identify critical components and establish their protection, emergency telephones, means for rerouting traffic to enable access of emergency vehicles, restricted access after span failure),

- Structural modifications (protection of lower parts of cables on cablestayed and suspension bridges, reinforce welds and bolted connections to ensure plastic capacity, use energy absorbing bolts to strengthen connections and reduce deformations, alternate load paths). 
It is noted that efficiency has to be assessed for all considered measures. For instance investments into motion sensors may decrease needs for the restriction of physical access. The control of access by guards is often ineffective since they can be intentionally distracted by secondary attacks. For blast attacks stand-off distance is a key parameter and can be hardly replaced by protective walls.

\section{Discussion}

Clearly, risk and cost-benefit considerations provide important insights into how security measures should perform, their effect on risk reduction, and their costeffectiveness [21]. A risk-averse attitude in which severe consequences are more weighed is commonly accepted in decision-making [27]. Despite this a riskneutral attitude is suggested in this study to obtain the most efficient option in terms of the expected life cycle cost.

Highway and road authorities and network managers may be more interested in the risks of a whole network rather than to the risks of an individual bridge. In this case the methodology proposed here should be extended similarly as proposed in [28].

\section{Concluding remarks}

Bridges in accidental situations represent the most vulnerable components of road networks. It is shown that decisions concerning selection of protective measures can be based on the risk-based optimisation of expected costs. Actions to be considered in the assessment may include normal and accidental (natural or manmade) loads and effects of human errors. Potential protective measures should include restriction of physical access, detection efforts, security planning and coordination, and construction modifications aiming to improve structural robustness. It appears that the probabilistic cost optimisation in conjunction with Bayesian networks provide an effective tool for decisions concerning appropriate protective measures for bridges endangered by various types of hazards.

\section{Acknowledgement}

The study is based on outcomes of the research project VG20122015089 supported by the Ministry of the Interior of the Czech Republic.

\section{References}

[1] Imam, B.M. and Chryssanthopoulos, M.K., Causes and Consequences of Metallic Bridge Failures. Structural Engineering International, 22(1), pp. 93-98, 2012.

[2] The European parliament and the Council, COUNCIL DIRECTIVE 2008/114/EC on the identification and designation of European critical 
infrastructures and the assessment of the need to improve their protection, Official Journal of the European Union: Brussels, pp. 8, 2008.

[3] Commission of the European Communities, Communication from the Commission on a European Programme for Critical Infrastructure Protection COM(2006) 786, pp. 13, 2006.

[4] Stewart, M., Risk-informed decision support for assessing the costs and benefits of counter-terrorism protective measures for infrastructure. International Journal of Critical Infrastructure Protection, 3(1), pp. 29-40, 2010.

[5] Bocchini, P. and Frangopol, D.M., A stochastic computational framework for the joint transportation network fragility analysis and traffic flow distribution under extreme events. Probabilistic Engineering Mechanics, 26(2), pp. 182-193, 2011.

[6] Holicky, M. and Sykora, M., Assessment of Flooding Risk to Cultural Heritage in Historic Sites. Journal of Performance of Constructed Facilities, 24(5), pp. 432-438, 2010.

[7] EN 1990, Eurocode - Basis of structural design, CEN: Brussels, pp. 87, 2002.

[8] EN 1991-1-7, Eurocode 1: Actions on structures - Part 1-7: General actions - Accidental actions, CEN: Brussels, 2006.

[9] SeRoN, Risk Assessment (Deliverable D500), The SeRoN Consortium, pp. 110, 2012.

[10] Stewart, M.G. and Melchers, R.E., Probabilistic Risk Assessment of Engineering Systems, Springer: Berlin, pp. 288, 1997.

[11] Melchers, R.E., Structural Reliability Analysis and Prediction, John Wiley \& Sons Ltd.: Chichester, England, pp. 437, 2001.

[12] JCSS, Risk Assessment in Engineering - Principles, System Representation \& Risk Criteria, Joint Committee on Structural Safety: Zurich, pp. 35, 2008.

[13] ISO 13824, Bases for design of structures - General principles on risk assessment of systems involving structures, ISO TC98/SC2: Geneve, Switzerland, pp. 43, 2009.

[14] Holicky, M., Optimisation of the target reliability for temporary structures. Civil Engineering and Environmental Systems, pp. 1-10, 2012.

[15] Faber, M.H. and Stewart, M.G., Risk assessment for civil engineering facilities: critical overview and discussion. Reliability Engineering \& System Safety, 80(2), pp. 173-184, 2003.

[16] Nishijima, K., Maes, M.A., Goyet, J. and Faber, M.H., Constrained optimization of component reliabilities in complex systems. Structural Safety, 31(2), pp. 168-178, 2009.

[17] Nielsen, T.D. and Jensen, F.V., Bayesian Networks and Decision Graphs, Springer: Berlin, pp. 448, 2007.

[18] Holicky, M., Markova, J. and Sykora, M., Forensic assessment of a bridge downfall using Bayesian networks. Engineering Failure Analysis, 30, pp. 1-9, 2013. 
[19] Holicky, M., Probabilistic risk optimization of road tunnels. Structural Safety, 31(3), pp. 260-266, 2009.

[20] Vrouwenvelder, T., Leira, B.J. and Sykora, M., Modelling of Hazards. Structural Engineering International, 22(1), pp. 73-78, 2012.

[21] Stewart, M.G. and Mueller, J., Terror, Security, and Money: Balancing the Risks, Benefits, and Costs of Critical Infrastructure Protection. Proceedings of Reliability Engineering Computing REC 2012, eds. M. Vořechovsky, V. Sadilek, S. Seitl, V. Vesely, R.1. Muhanna and R.1. Mullen, Brno University of Technology: Brno, pp. 513-533, 2012.

[22] Vrouwenvelder, T., Stochastic modelling of extreme action events in structural engineering. Probabilistic Engineering Mechanics, 15(1), pp. 109-117, 2000.

[23] JCSS, JCSS Probabilistic Model Code, Joint Committee on Structural Safety: Zurich, 2011.

[24] Netherton, M.D. and Stewart, M.G., Blast load variability and accuracy of blast load prediction models. International Journal of Protective Structures, 1(4), pp. 543-570, 2010.

[25] Thoft-Christensen, P., Life-cycle cost-benefit (LCCB) analysis of bridges from a user and social point of view. Structure and Infrastructure Engineering, 5(1), pp. 49-57, 2009.

[26] Berrick, C.A., Highway Infrastructure: Federal Efforts to Strengthen Security Should be Better Coordinated and Targeted on the Nation's Most Critical Highway Infrastructure, DIANE Publishing Company: 2009.

[27] Cha, E.J. and Ellingwood, B.R., Risk-averse decision-making for civil infrastructure exposed to low-probability, high-consequence events. Reliability Engineering \& System Safety, 104 pp. 27-35, 2012.

[28] Bocchini, P. and Frangopol, D.M., Generalized bridge network performance analysis with correlation and time-variant reliability. Structural Safety, 33(2), pp. 155-164, 2011. 